
\title{
CIÊNCIA'NATURA
}

\section{Comportamento do Dióxido de Nitrogênio Sobre a Região Nordeste do Brasil e sua Relação com Variáveis Meteorológicas}

\author{
Behavior of Nitrogen Dioxide On the Northeast Region of Brazil and Its Relationship with \\ Meteorological Variables \\ Ismael Guidson Farias de Freitas ${ }^{1}$, José Carlos da Silva Santos², Ericka Voss Chagas Mariano ${ }^{3}$ e Glauber Lopes \\ Mariano $^{3}$ \\ ${ }^{1}$ Mestrando em Meteorologia, Instituto de Ciências Atmosféricas- UFAL, Maceió- AL, Brasil \\ ismael.guidson@gmail.com \\ ${ }^{2}$ Graduando em Meteorologia, Instituto de Ciências Atmosféricas- UFAL, Maceió- AL, Brasil \\ josecarlos220597@gmail.com \\ ${ }^{3,4}$ Professor(a) de Meteorologia, Instituto de Ciências Atmosféricas- UFAL, Maceió- AL, Brasil \\ erickavoss@gmail.com/glaubermariano@gmail.com,
}

\begin{abstract}
Resumo
A emissão de poluentes tem se tornado um dos assuntos mais debatidos nos últimos anos, devido ao aumento de fontes emissoras fixas como industrias e móveis como os veículos automóveis. No Nordeste Brasileiro as altas concentrações de poluentes se dão, principalmente, nos grandes centros urbanos, onde um destes poluentes emitido é o dióxido de nitrogênio. Uma vez observada altas concentrações desse poluente na atmosfera o mesmo podem causar efeitos na saúde humana e influencia ainda na formação de chuva ácida. Assim, o objetivo deste trabalho foi investigar e quantificar a coluna total do gás dióxido de nitrogênio sobre a região nordeste do Brasil baseado nos dados oriundos do sensor OMI (Ozone Monitoring Instrument) entre o periodo de 2005 a 2015, através de técnicas estatísticas voltadas a análise gráfica e espacial. Identificou-se variações consideráveis do poluente na área de estudo, destacando-se valores elevados em Salvador, Teresina e Fortaleza, onde as mesmas apresentaram ainda resultados significativos de correlação com variáveis meteorológicas. Os resultados apresentaram que Salvador teve concentrações de até 3,5 E+15(1/Cm $\left.{ }^{2}\right)$ na coluna total de NO2, enquanto Teresina e Fortaleza, tiveram máximo de 3,0 E+15 $\left(1 / \mathrm{Cm}^{2}\right)$ e 2,6 E+15 $\left(1 / \mathrm{Cm}^{2}\right)$, respectivamente. Portanto, as variações foram diferentes nas cidades.
\end{abstract}

Palavras-chave: poluição atmosférica; química da atmosfera; sensoriamento remoto; dióxido de nitrogênio; OMI.

\begin{abstract}
The emission of pollutants has become one of the subjects most debated in the last years, due to the increase of fixed emitting sources like industries and movable like the motor vehicles. In the Northeast of Brazil, the high concentrations of pollutants occur mainly in large urban centers, where one of these pollutants is emitted nitrogen dioxide, which is a highly polluting gas. Once observed high concentrations of this pollutant in the atmosphere can cause effects on human health and also influence the formation of acid rain. Thus the objective of this work was to investigate and quantify the total column of nitrogen dioxide gas over the northeastern region of Brazil based on data from the OMI sensor (Ozone Monitoring Instrument) between 2005 and 2015, through statistical techniques for analysis graphic and spatial. Significant variations of the pollutant were identified in the study area, highlighting Salvador, Teresina and Fortaleza, where they showed significant correlation results with meteorological variables. The results showed that Salvador had concentrations of up to $3.5 \mathrm{E}+15\left(1 / \mathrm{m}^{2}\right)$ in the total NO2 column, while Teresina and Fortaleza had a maximum of $3.0 \mathrm{E}+15\left(1 / \mathrm{m}^{2}\right)$ and 2,6 E+15 $\left(1 / \mathrm{m}^{2}\right)$, respectively. Therefore, the variations were different in the cities.
\end{abstract}




\section{Introdução}

Com o crescimento populacional e o desenvolvimento industrial ocorridos nas últimas décadas, diversas fontes de poluentes antropogênicas vem surgindo, resultando em diversas mudanças globais no ambiente e principalmente na composição atmosférica. Nos grandes centros urbanos do Nordeste Brasileiro um dos principais causadores da emissão de poluentes é o grande trafego de veículos automotores nas vias públicas que são responsáveis por emitir poluentes primários como o NO2 (Dióxido de Nitrogênio) (FERNANDES MANOEL FILHO DA SILVA, 2007).

O NO2 é produzido a partir da reação química com a oxidação de átomos de nitrogênio contidos no combustível e na atmosfera, processo que varia dependendo da temperatura (ARAÚJO, NAHIRNY e FELIX, 2014), além de influências das condições meteorológicas que exercem um papel fundamental na qualidade do ar as variáveis meteorológicas como velocidade do vento, direção do vento e precipitação guiam a dispersão de poluentes e a sua deposição (MOREIRA,TIRABASSI e MORAES, 2008)

Devido as emissões o clima urbano vem sendo alterado com o passar dos anos o que significa que a emissão de poluentes na atmosfera é uma variável importante, pois controlar a concentração dessa variável é vital para a saúde humana, e por se tratar de um gás tóxico a altas concentrações (CORREIA, 2011). Quando inalado, o NO2 atinge as porções mais periféricas do pulmão por conta da sua baixa solubilidade (BRAGA, 2001), provocando problemas respiratórios. Sendo assim, estudar, quantificar e analisar a variação da coluna total do dióxido de nitrogênio sobre a região do Nordeste Brasileiro é imprescindível para melhor compreender e realizar o monitoramento destes poluentes.

Por se tratar de uma extensa área e existir falta de investimento para a aquisição de estações de monitoramento da qualidade do ar no Nordeste Brasileiro, o uso de satélites tem sido fundamental neste tipo de estudo, uma vez que é possível realizar o monitoramento em grande expansão espacial e em pequeno espaço temporal. Desse modo, através dos produtos obtidos através do Satélite Aura é possível gerar imagens espectrais capturadas no comprimento de onda na região do visível permitindo quantificar o $\mathrm{NO} 2$ presente na atmosfera e saber os locais com maiores concentrações do poluente anteriormente citado.

Por fim, o objetivo deste trabalho é quantificar as colunas totais dos gases dióxido de nitrogênio, analisar estatisticamente os dados de coluna total de NO2, identificar a variação espacial e temporal do gás e avaliar possíveis tendências mensais e sazonais, indicando se há relação com algumas variáveis meteorológicas e identificar as principais áreas da região Nordeste com altas concentrações.

\section{Materiais e Métodos}

Através do site https://giovanni.gsfc.nasa.gov/giovanni/, da agência espacial americana (NASA) obteve-se os dados de dióxido de nitrogênio durante o período de 2005 a 2015. Os dados foram gerados pelo sensor OMI coletados com o comprimento de onda 0,35 a $0,5 \mu \mathrm{m}$ correspondente a Banda 3 , e utilizados para produzir uma série temporal ao longo dos anos e fazer uma análise de tendências da concentração de dióxido de nitrogênio da coluna total para as mesmas regiões em períodos desejados. Afim de se obter um melhor resultado, optou-se por dividir a região nordeste do Brasil em quadrantes de arestas $1^{\circ}$ por $1^{\circ}$.

Os gráficos foram gerados para mostrar a variação mensal, anual e interanual da concentração da coluna total do dióxido de nitrogênio sobre a região Nordeste do Brasil, no período de 2005-2015, onde também foi realizada a correlação das concentrações do poluente analisado, com variáveis meteorológicas afim de se discutir possíveis influências. 
Os dados das variáveis meteorológicas foram obtidos através do sistema de coleta utilizado pelo Instituto Nacional de Meteorologia (INMET), que possui estações de sondagem de ar superior (radiossonda), estações meteorológicas de superfície e a maior rede de estações automáticas da América do Sul, cujas informações são disponibilizadas por meio do endereço https://www.inmet.gov.br.

\section{Resultados e Discussões}

\subsection{Variação anual do NO2}

Para este estudo foram feitas análises para todas as capitais dos estados do nordeste brasileiro, porém o enfoque se deram nas capitais Salvador- BA, Teresina- MA e Fortaleza- PI, por se tratar das maiores cidades do Nordeste em termo de população e densidade demográfica.

Levando em conta o intervalo de tempo 2005-2015, Salvador (Figura 1a), apresentou períodos onde a coluna total oscilou, dando destaque para o aumento durante 2011-2013, onde atingiu o seu valor máximo. Entre 2008-2011, observou-se diminuição das colunas totais (Figura 1a). Em relação à cidade de Fortaleza (Figura 1c), notou-se um período prolongado de aumento nas colunas totais (2004-2011), porém sem muita divergência nos dados. Para essa capital, bem como a de Teresina, foi observado tendência de aumento das colunas totais entre 2011-2012, onde Fortaleza atinge seu valor máximo. No período de 2012-2014, para Fortaleza foi constatada atenuação dos dados analisados. De todos os dados destacados, Fortaleza foi o local em que houve menos variação ao longo dos anos (Figura 1c). Na Figura 1(d), que apresenta a variação média da coluna total de dióxido de nitrogênio para toda a área do nordeste, a variação entre o período estudado se deu semelhante a cidade de Teresina, apresentando comportamento similar entre os picos e decréscimos. Foi observado, também, que a coluna total do poluente na região Nordeste do Brasil, no período de 2005-2015, se manteve variando entre os extremos 2,1E+15 e 2,7E+15 1/(Cm².

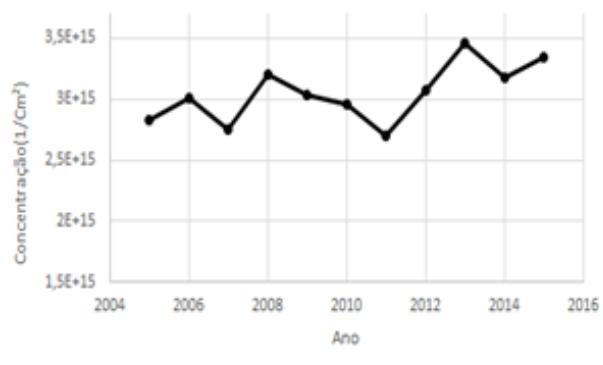

(a)

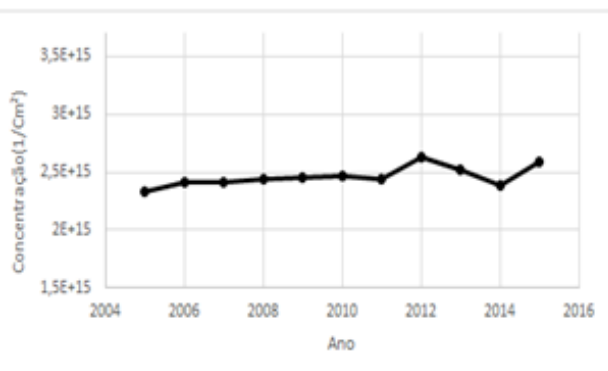

(c)

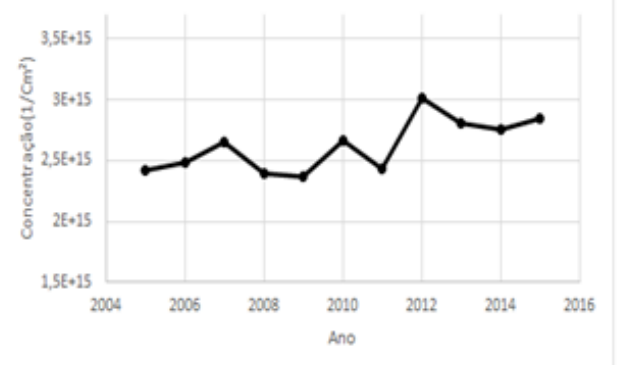

(b)

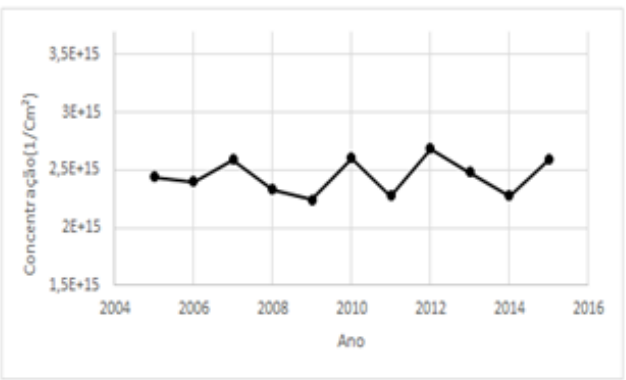

(d)

Figura 1 - Variação anual do dióxido de nitrogênio para as capitais de Salvador (a), Teresina (b), Fortaleza (c) e região Nordeste (d) detactadoc nalo concor OMI duranta a naríndo $9005-9015$ 
Foram selecionadas na Figura 1d os pontos com maiores valores da coluna total de dióxido de nitrogênio, correspondentes os anos de 2010 e 2012 (anos com maiores valores de concentração observado) e após foi plotado o gráfico de distribuição espacial para todo o nordeste do Brasil.

Apesar dos valores da média no ano de 2010 e 2012 serem próximos, foram observadas maiores variações espaciais da variável sobre o Nordeste brasileiro (Figura 2a e Figura 2b), onde na figura 2 (a) na região central do estado do Maranhão, sul do Piaú e oeste da Bahia, apresentou núcleo com valores intensos (Figura 2a), reduziu no ano de 2012 (Figura 2b), no qual os núcleos ficaram presente apenas na região central do Piauí, surgindo no oeste da Bahia outro núcleo com valores altos, porém menor distribuído espacialmente. Já os núcleos que encontravam- se ao norte da Bahia, sul do Ceará, na divisa entre os estados da Paraíba e Pernambuco, e em Salvador (leste da Bahia) (Figura 2a), ocorreu a diminuição nos valores e na distribuição espacial no ano de 2012(Figura 2b).
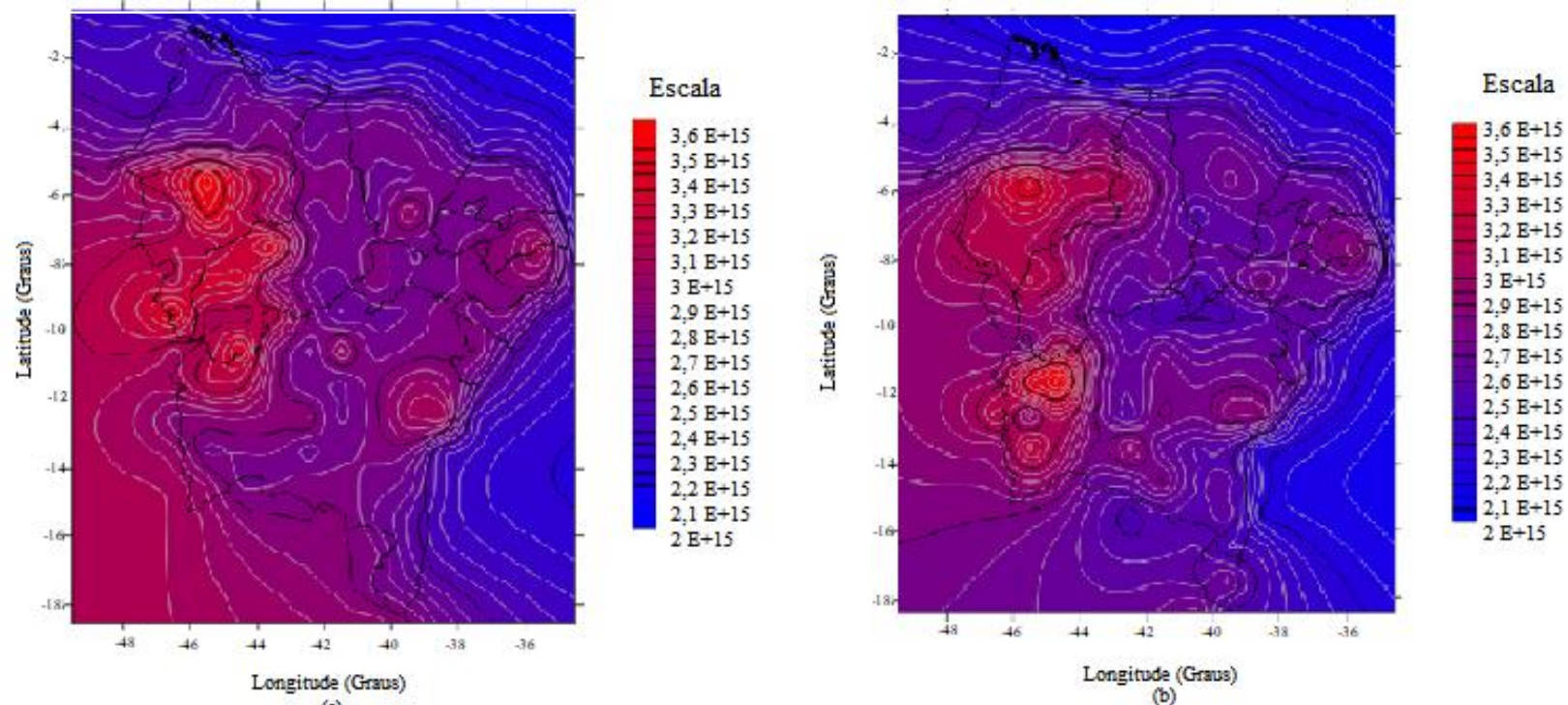

Figura 2 - Dados da variação anual espacial do dióxido de nitrogênio em relação aos anos de 2010 (a) e 2012 (b) para o Nordeste brasileiro.

\subsection{Variação mensal do NO2}

A cidade de Salvador, de janeiro a junho (período compreendido entre os solstícios de verão e inverno), apresentou pouca variação na concentração de dióxido de nitrogênio (Figura 3a). Contudo, houve tendência de aumento dos dados de junho a novembro (do início do inverno até um pouco antes do fim da primavera), onde a concentração atinge seu valor mínimo e máximo em junho e novembro, respectivamente. Para a cidade de Fortaleza, foi constatada diminuição da coluna total entre janeiro e fevereiro, além de tendência de aumento na linha temporal limitadas pelos meses de fevereiro e setembro (entre o verão e o início da primavera), onde atingiu seu valor mínimo e máximo em fevereiro e setembro respectivamente. De setembro a dezembro, houve diminuição da concentração do poluente estudado (Figura 3b). Durante o intervalo correspondido entre janeiro e março, para a cidade de Teresina (Figura 3c), foi notado decréscimo na variação das colunas totais, porém, entre março e maio, não houveram diferenças consideráveis nos valores assumidos pela variável em estudo, ou seja, não apresentaram diferenças significativas entre si. Foi observado diminuição da concentração do poluente em estudo, de setembro a dezembro. Em setembro, a concentração atingiu seu valor máximo. Na Figura 3(d) podemos observar a variação mensal da concentração do dióxido de nitrogênio durante o período de 2005-2015 sobre a região Nordeste. Entre janeiro e maio, observou-se 
diminuição da variação do NO2, e consequentemente a variável atingiu seu menor valor. Houve tendência de aumento entre maio e setembro (início da primavera), onde atingiu o maior valor da variável. Para o espaço de tempo correspondido entre setembro e dezembro, foi constatada diminuição das colunas totais analisadas.

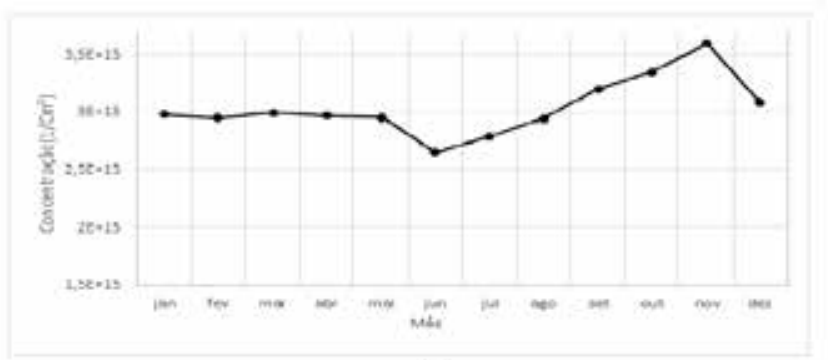

(a)

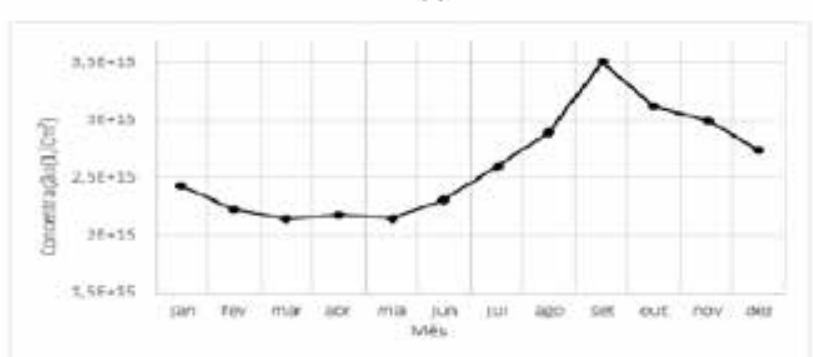

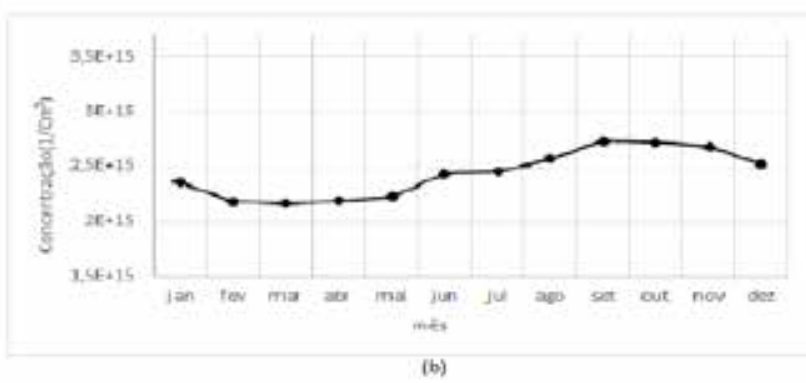

(b)

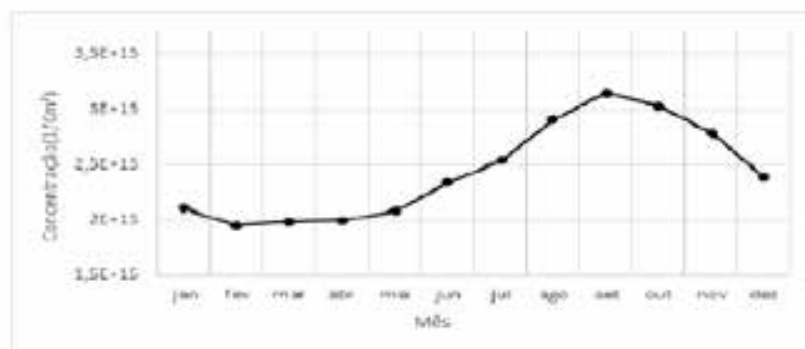

Figura 4- Média mensal do dióxido de nitrogênio sensor OMI durante o período 2005-2015 para a cidade de Salvador (a), Fortaleza (b), Teresina (c) e região Nordeste (d).

\subsection{Correlação do NO2 com Variáveis Meteorológicas}

A maioria dos dados de correlação analisados, possuem um grau de irrelevância, por esse motivo, optou-se por representar apenas as capitais cuja concentração do poluente relacionado indicou maiores influências das variáveis meteorológicas (temperatura, precipitação e velocidade média do vento) no período compreendido entre 2005 e 2015 . A concentração do dióxido de nitrogênio para as cidades de Teresina, Salvador e Fortaleza e os dados de precipitação obtidos no período de 2005-2015, apresentaram uma forte correlação negativa, onde foi obtido os valores -0,7184, 0,7009 e -0,9321 respectivamente, significando que a reta de regressão linear possui um bom ajustamento entre os dados de forma inversamente proporcional (Figuras 5a, 5b e 5c). Além disso, como era esperado, com o aumento da precipitação ocorreram diminuições nas concentrações das colunas totais das regiões destacadas. Para a temperatura notou-se forte positividade no valor do coeficiente de determinação para as cidades de Teresina e Fortaleza, sendo eles 0,8426 e 0,7228, na devida ordem. Porém, em Salvador esse valor foi menor (0,2788), indicando pouca qualidade no ajustamento da linha de tendência (Figuras $5 \mathrm{~d}, 5 \mathrm{f}$ e $5 \mathrm{~g}$ ). Com o aumento da temperatura, percebe-se, de acordo com as figuras 6(b), 6(e) e 6(h), acréscimos nas concentrações das colunas totais do NO2. Os maiores valores obtidos pelo coeficiente de determinação foram provenientes da correlação entre o NO2 e a velocidade do vento (Figuras 5h, $5 \mathrm{i}$ e $5 \mathrm{j}$ ), indicando melhor ajuste da linha de regressão. Os valores obtidos através dessa correlação foram 0,9109 para Teresina, 0,6614 em Salvador e 0,8957 associado a Fortaleza. 


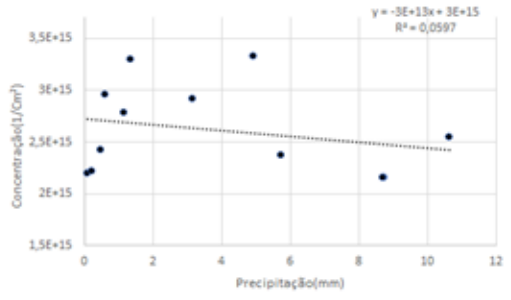

(a)

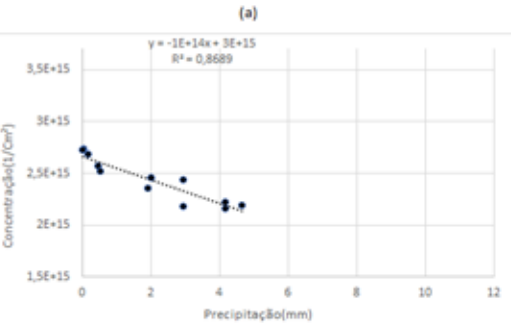

(d)

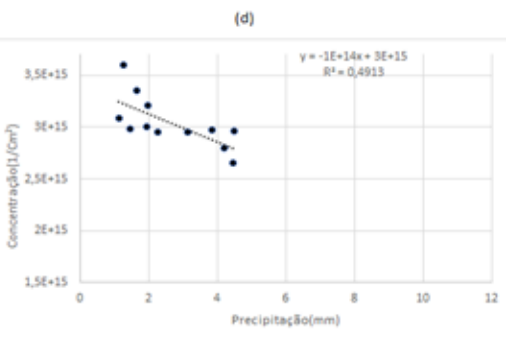

$(8)$

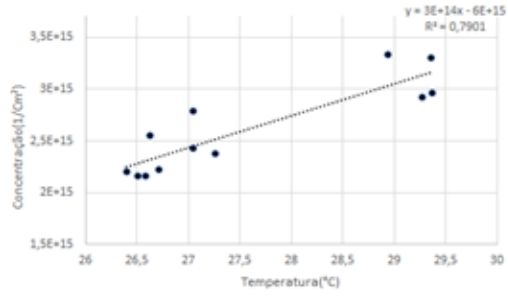

(b)

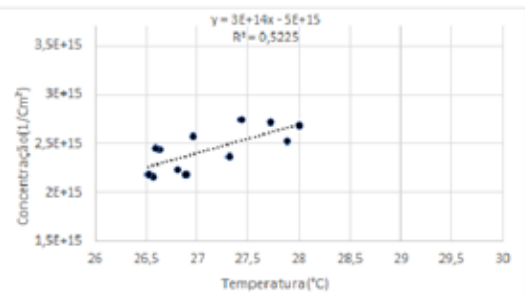

(e)

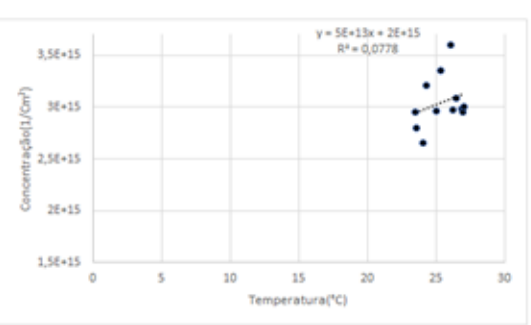

(h)
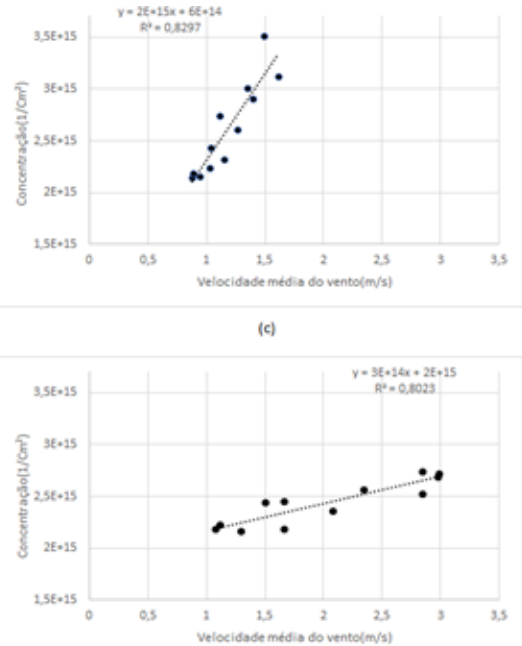

(1)

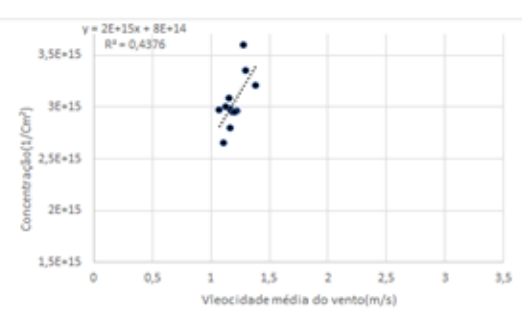

Figura 6- Correlação das colunas totais do NO2 com variáveis meteorológicas (Precipitação, Temperatura e Velocidade do vento), durante o período 2005-2015 para as capitais de Teresina (5a, 5b, 5c), Fortaleza (5d,5e,5f) e Salvador (5g,5h,5i).

\section{Conclusões}

Na região Nordeste do Brasil as capitais com maiores valores na coluna total do dióxido de nitrogênio entre o período de 2005-2015 foram Salvador, Teresina e Fortaleza. De janeiro a abril, nessas capitais, ocorreu diminuição da variação mensal da coluna total do poluente estudado. Este fato pode ser fundamentado pelo aumento de temperatura sobre essa região ser maior durante esse período, convertendo mais rapidamente NO2 em ozônio. No decorrer de setembro e dezembro, foi constatada atenuação dos dados mensais do dióxido de nitrogênio para a capital de Teresina, Fortaleza e região Nordeste de forma geral.

A partir da análise feita tomando como base a correlação entre as concentrações das colunas totais das capitais da região em estudo e as variáveis meteorológicas temperatura, precipitação e velocidade do vento, notou-se valores negativos referentes aos coeficientes de determinação entre Fortaleza e os dados de precipitação representados neste documento. Esse resultado mostra pouco ajustamento da linha de regressão, além das variáveis possuírem uma correlação negativamente forte entre si, mostrando que houve influência da precipitação sobre o NO2 durante o período de tempo analisado, favorecendo a diminuição da concentração do poluente estudado. Todas as capitais destacadas apresentaram uma forte correlação com as variáveis meteorológicas, exceto Salvador, onde seu coeficiente de determinação com a Temperatura foi positivamente fraco durante 2005-2015. 


\title{
Referências
}

ANNE DOUGLASS, OMI Ozone Monitoring Instrument, 2004. Disponível em:< https://aura.gsfc.nasa.gov/omi.html> Acesso em: 02 de mar. 2017.

ARAUJO, A. S. D; NAHIRNY, E. P.; FELIX, E. P. Medidas de Dióxido de Nitrogênio na Atmosfera de Ambientes Internos e Externo. In: 37a Reunião Anual da SBQ, 2014, Natal-RN.

FERNANDES, R. C.; MANOEL FILHO, F. N.; DA SILVA, M. Análise das Concentrações de NOX, NO, $\mathrm{NO}_{2}, \mathrm{O}_{3}$ no Centro Urbano de Maceió, AL. Ciência e Natura2007, v. 29, p. 229.

CORREIA, J. G. A. Estudo Preliminar de $\mathrm{NO}_{2}$ na Atmosfera da Cidade de João Pessoa. 2011. 54 f. Dissertação (Mestrado em Química) — Universidade Federal da Paraíba, Paraíba, 2011.

BRAGA, A. et al. Poluição atmosférica e Saúde Humana. Revista USP,2001,n.51, p. 58-71.

MOREIRA, D. M.; TIRABASSI, T.; MORAES, M. R. Meteorologia e Poluição Atmosférica. Ambiente \& Sociedade,2008,v. 11, n. 1, p.1-13.

\section{Ismael Guidson Farias Freitas}

Instituto de Ciências Atmosféricas, UFAL, Maceió - AL, Brasil Mestrando em Meteorologia, E-mail: ismael.guidson@gmail.com

José Carlos da Silva Alves

Instituto de Ciências Atmosféricas, UFAL, Maceió - AL, Brasil Graduando em Meteorologia, E-mail: josecarlos220597@gmail.com

Ericka Voss Chagas Mariano

Instituto de Ciências Atmosféricas, UFAL, Maceió - AL, Brasil Professora em Meteorologia, E-mail: erickavoss@gmail.com

\author{
Glauber Lopes Mariano
}

Instituto de Ciências Atmosféricas, UFAL, Maceió - AL, Brasil Professor em Meteorologia , E-mail: glaubermariano@gmail.com 